\title{
The Effect of Cerebrospinal Fluid in Collagen Guide Channel on Sciatic Nerve Regeneration in Rats
}

\author{
Gholam Hossein FARJAH ${ }^{1}$, Mohammad Amin DOLATKHAH², Bagher POURHEIDAR ${ }^{1}$, Behnam HESHMATIAN ${ }^{3}$ \\ ${ }^{1}$ Urmia University of Medical Sciences, Faculty of Medicine, Neurophysiology Research Center, Department of Anatomy, Urmia, Iran \\ ${ }^{2}$ Urmia University of Medical Sciences, Faculty of Medicine, Graduated from Department of Anatomy, Postgraduate Masters from \\ Department of Anatomy, Urmia, Iran \\ ${ }^{3}$ Urmia University of Medical Sciences, Faculty of Medicine, Neurophysiology Research Center, Department of Physiology, Urmia, Iran
}

\section{ABSTRACT}

AIM: The aim of this study was to evaluate the effect of cerebrospinal fluid (CSF) in nerve regeneration across the collagen guide channel in comparison with autograft.

MATERIAL and METHODS: Forty adult male rats (250-300 g) were randomized into (1) collagen channel+CSF, (2) collagen channel+normal saline (NS), (3) autograft, and (4) sham surgery groups. The left sciatic nerve was exposed and a $10 \mathrm{~mm}$ nerve segment was cut and removed. In the collagen groups, the proximal and distal cuts ends of sciatic nerve were telescoped into the nerve guides and CSF or NS injected into collagen conduit. In the autograft group, the $10 \mathrm{~mm}$ nerve segment was turned backwards and used an autologous nerve graft. All animals were evaluated by sciatic functional index (SFI) and electrophysiology, histology, and immunohistochemistry testing.

RESULTS: The improvements in SFI since the beginning of the last evaluation in experimental groups were measured. On days 49 and 60 post-operation, the mean SFI of the collagen+CSF group was significantly greater than the autograft group $(P<0.05)$. On day 90 , the mean nerve conduction velocity (NCV) of the collagen+CSF group was greater than autograft group $(P<0.05)$. The number of myelinated fibers in the collagen+CSF group was significantly greater than that of the collagen $+N S$ group at day $90(P<0.05)$.

CONCLUSION: CSF in collagen nerve guide channel effectively enhances nerve regeneration and promotes functional recovery in injured sciatic nerve of rats.

KEYWORDS: Sciatic nerve, Collagen, Nerve guide channel, Cerebrospinal fluid, Rat

\section{INTRODUCTION}

$\mathrm{F}$ Tor peripheral nerve repair, nerve autografts have always been considered as the "gold standard" for the restoration of structural and functional nerve regeneration (34).

Autograft has several disadvantages, including the need for an extra-incision, loss of donor nerve function, mismatch in size between the donor nerve and the injured nerve, and a limited availability of donor nerve (33). To solve these problems, nerve guide channels (NGCs) have been made to bridge the nerve gaps by inserting the severed nerve stumps into the two ends of the canal (16). Some natural and synthetic substances can be shaped in structures that are able to promote nerve regeneration for example: eggshell membrane (12), silicone (31), human amnion layer (22), collagen (18), collagen-Chitosan (36).

Collagen-based biomaterials have been extensively studied as a promising nerve guide (7). Since collagen is a natural material, it shows excellent biocompatibility, insignificant immunogenicity, and high bio-absorbability (27). 
Collagen is able to promote nerve regeneration (2). Collagen as a major part of the extracellular matrix is broadly utilized as cloth in various surgical prostheses and was previously also used for nerve repair $(7,20)$.

The use of neurotrophic factors in the care and survival of peripheral neuronal cells has been the topic of numerous studies (29). Cerebrospinal fluid (CSF) has been reported to contain nerve growth factor (NGF) and transforming growth factor alpha (TGF-alpha) (35). Although the role of the CSF during embryogenesis is just starting to be studied, several recent papers suggest an important role for CSF in brain development. Miyan et al. have shown that rat cortical cells are viable and proliferate in CSF, and recent studies have begun to test discrete signaling factors that may regulate neurogenesis (21).

Growing evidence suggests that CSF plays an important role not only in brain development but also in survival and proliferation of neuroectodermal stem cell (13). A previous study showed that adult CSF facilitates gliogenesis from the fetal rat neural stem cell (5). Despite the easy availability of collagen and CSF and its use in traumatic lesions of various tissues, their effects on the peripheral nerves are not determined.

The purpose of this experiment is to evaluate the effect of CSF in the collagen channel by functionally, electrophysiology, histology and immunohistochemistry testing in comparison with autograft.

\section{MATERIAL and METHODS}

\section{Animals}

Forty adult male Sprague-Dawley rats (250 to $275 \mathrm{~g}$ ), were divided randomly into four equal groups: sham surgery, collagen channel+normal saline (NS), collagen channel+CSF, and nerve autograft. This study was approved by the ethical committee of Urmia University of Medical Sciences. The left sciatic nerve was used as experimental side and the other side to serve as the control.

\section{Preparation of Rat-Tail Collagen}

Collagen I was extracted from rat-tail tendons by modification of the previously published method (15). Briefly, tendons was excised from the tails of Sprague-Dawley rats and washed in ethanol $70^{\circ}$ for $45 \mathrm{~min}$. They were transferred to acetic acid (200 $\mathrm{\mu l}$ glacial per $1 \mathrm{~g}$ tendon in $100 \mathrm{ml}$ demonized water), then stirred for 7 days at $4^{\circ} \mathrm{C}$. The resulting suspension was centrifuged at $3000 \times \mathrm{g}$ for 30 minutes at $4^{\circ} \mathrm{C}$. The supernatant was removed, and averaging the $3 \%$ collagen solution was obtained. In order to sterilize the collagen I, chloroform (10\% of the volume of collagen) was layered beneath the collagen solution and allowed to rest for $24 \mathrm{~h}$ at $4{ }^{\circ} \mathrm{C}(28)$. The solution was immersed in demonized water, $\mathrm{pH}$ to 7.4 with $\mathrm{NaOH}$ and PBS on ice and mixed thoroughly with a pipette (9).

\section{Preparation of Collagen Guide Channel}

Under laminar air flow, the collagen channels were prepared by a modification of the previously published technique (19).
Briefly, a Teflon tube, with an external diameter of $1.6 \mathrm{~mm}$, was immersed in collagen solution and dried at room temperature. This step was repeated 20 times. Then, it was subjected to thermal dehydration at $105^{\circ} \mathrm{C}$ for 24 hours. Finally, the Teflon tube was removed and a collagen nerve guide $(1.6 \mathrm{~mm}$ in inner diameter and $12 \mathrm{~mm}$ in length) remained (Figure 1A, B).

\section{Collection of CSF from Cisterna Magna}

Ten adult male Sprague-Dawley rats (250-275 g) were anesthetized with ketamine and xylazine (50 and $10 \mathrm{mg} / \mathrm{kg}$ per body weight, respectively). CSF from cisterna magna was collected by the previously published method (11). Briefly, the anesthetized animals was then mounted on stereotaxic device (David Kopf, California, USA). The atlanto-occipital membrane was exposed and a 33-gauge collecting needle was used for aspiration (11). After collection, the CSF samples was centrifuged for $10 \mathrm{~min}$ at $2000 \times \mathrm{g}$, and the supernatant was immediately stored at $-80^{\circ} \mathrm{C}$ until injected into collagen channels (6) (Figure 2A, B).

\section{Surgical Procedure}

Under general anesthesia with intraperitoneal ketamine (90 milligram $/ \mathrm{kg}$ ) and xylazine (10 milligram/kilogram), the left sciatic nerve was exposed. A $10 \mathrm{~mm}$ nerve segment was removed proximal to the tibial and the peroneal nerve bifurcation. In the collagen channel groups, the transected proximal and distal stumps were being inserted into the nerve guide and two 10-0 nylon suture was placed at each end to fix the tube in place. Before inserting the distal stump, the channels were filled with NS or CSF. In the autograft group, the $10 \mathrm{~mm}$ nerve segment was turned and given as an autologous nerve graft. The muscle and the skin were closed (Figure 3 ).

\section{Functional Track Analysis}

Animal were tested one day before surgery and on the $7^{\text {th }}$, $21^{\text {st }}, 35^{\text {th }}, 49^{\text {nd }}, 60^{\text {th }}$ and $90^{\text {th }}$ day post-operation. Indian ink was applied to the plantar surface of the hind feet to cover the entire anatomical regions. The animal was permitted to walk down the track, leaving its hind footprints on the paper. The footprints of both the operated and unoperated limbs were used to calculate the sciatic functional index (SFI) as described by Bain et al. (3).

\section{Electrophysiology}

At the 30 and 90 days after operation, five animals from each group were subjected to electrophysiological studies using Narco bio-system (USA). During the test, each rat's body temperature was kept constant between $36.5-37^{\circ} \mathrm{C}$ by mean of a temperature control unit (Narco, USA). Under intraperitoneal (i.p.) urethane anesthesia $(1 \mathrm{~g} / \mathrm{kg})$, the left sciatic nerve (operated side) was re-exposed. Stimulating electrodes were positioned in the proximal and the distal trunk of the grafted nerve and recording electrodes were located in the belly of the gastrocnemius muscle. The physiologic parameters (Latency and amplitude of compound action potentials) were obtained, then the nerve conduction velocity (NCV) was determined.

\section{Histology}

Immediately after electrophysiological evaluation, middle 

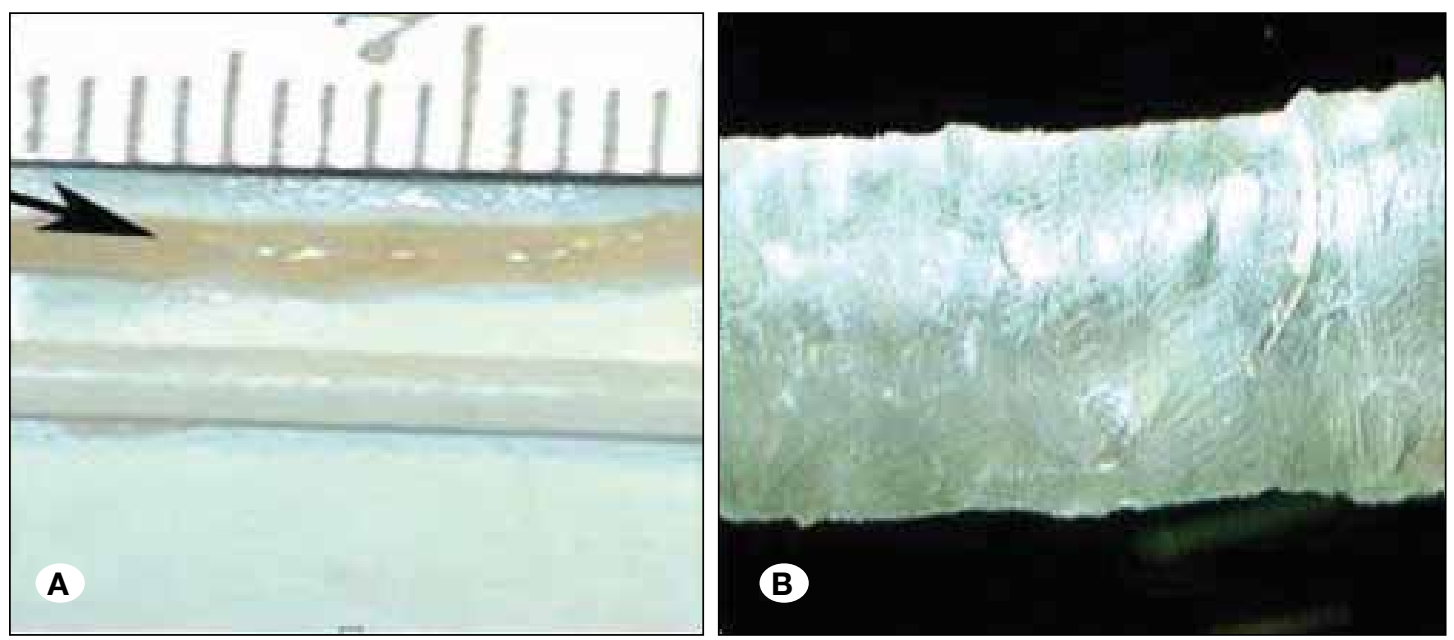

Figure 1:

A) Representative photograph of rat's sciatic nerve (Arrow) and nerve guide channel made from collagen I. The Teflon tube was immersed in collagen solution, dehydrated, removed, and the collagen channel remained. B) Photomicrograph of the collagen guide channel.
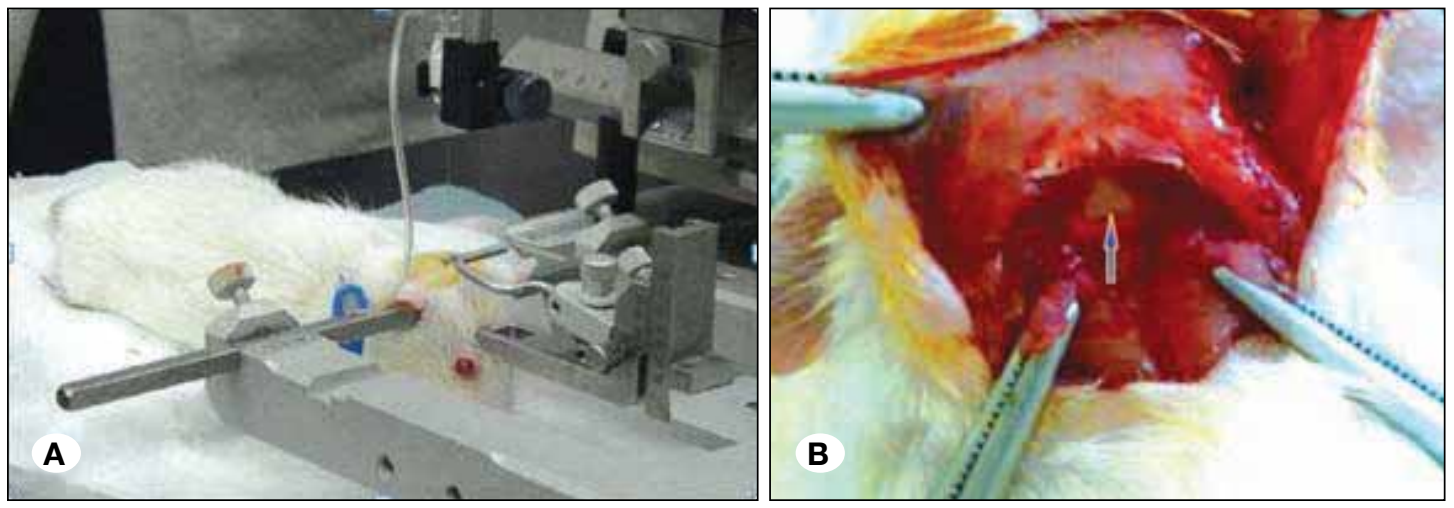

Figure 2: Positioning of the animal for collection of CSF from cisterna magna. A) Lateral view of stereotaxic setup. B) Exposure of the atlanto-occipital membrane. Arrow indicates the point of needle insertion.

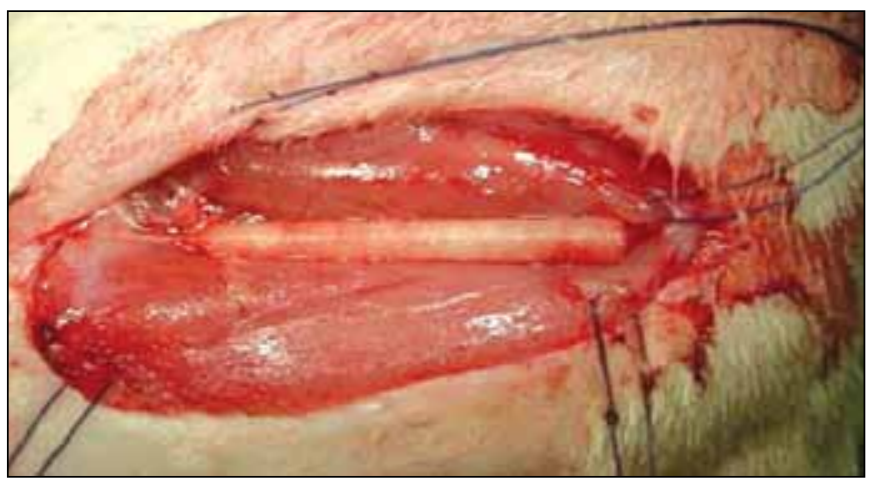

Figure 3: Implanted collagen guide channel on the sciatic nerve site. A $10 \mathrm{~mm}$ sciatic nerve segment was removed, and the transected proximal and distal stumps were inserted into the nerve guide.

cable on study groups were removed. The samples were fixed at $10 \%$ buffered formalin, dehydrated, embedded in paraplast paraffin blocks, cut transversely $5 \mu \mathrm{m}$, and stained with toluidine blue. Total myelinated fiber counts and myelinated fiber diameter measurements were performed in each nerve cross-section with the aid of (OLYSIA Biorefort, Olympus, Japan) morphometric analysis system. All measurements were made by a single, skilled observer.

\section{Immunohistochemistry}

Specimens prior to immunohistochemistry were post-fixed with 4\% Paraformaldehyde for 2 hours, embedded in paraffin and cut into $5 \mu \mathrm{m}$ thick sections. In this subject field, anti S-100 (Dako, 1:200 dilution) was employed as a marker for myelin sheath. According to the instructions of immunohistochemical staining kits, non-specific immunoreactions were blocked, sections were incubated in S-100 protein antibody solutions for 1 hour at room temperature. They were rinsed three times with phosphate buffered saline (PBS) and incubated with biotinylated anti-mouse, rabbit IgG solution for 1 hour at room temperature. The sections were therefore washed with PBS for 5 minutes, 3 times in all. Horseradish peroxidase-labelled secondary antibody solution was added to the sections, and incubated for 10-20 minutes at room temperature and washed with PBS for 5 minutes, 3 times in all. In the end, the sections were fully washed under running tap water and counterstained and mounted and examined under a light microscope.

\section{Statistical Analysis}

Statistical analysis was done by a mixed-design (within- and between-group comparisons) ANOVA were computed with 95\% confidence intervals using the SPSS software (version 16.0). All data are shown as mean \pm SD and, $p<0.05$ was considered statistically significant. 


\section{RESULTS}

All of the rats tolerated the operation. At 49 and 60 days postoperation, the mean SFI values were $-41.64 \pm 3.73,-38.04 \pm 5.11$ for the collagen+CSF group, and $-59.89 \pm 4.78,-49.45 \pm 6.21$ for the autograft group, respectively $(p<0.05)$. In addition, collagen+CSF and autograft groups were statistically different from the control group $(p<0.05)$. There were no statistically significant differences between collagen+CSF and autograft SFI values at 90 days post-operation (Figure 4).

At the $90^{\text {th }}$ day, the mean nerve conduction velocities (NCV) were $39.7 \pm 3.53 \mathrm{~m} / \mathrm{Sec}, 30.05 \pm 4.71$ for the collagen $+\mathrm{CSF}$ and autograft groups, respectively. The mean NCV of the collagen+CSF group was higher than of the autograft group, and the difference was statistically significant $(p<0.05)$. At the same time, the mean amplitude (AMP) values in the collagen+CSF and autograft groups were 5.72 $\pm 0.28 \mathrm{MV}$ and 4.76 $\pm 0.98 \mathrm{MV}$, respectively. The difference was not statistically significant ( $p>0.05)$ (Table I).

In the collagen+CSF group, the nerve cables contained fascicles of axons. The neural tissue was centrally located in the guide channel and surrounded by an epineurium composed of several layers of circumferentially arranged fibroblast and collagen fiber layers. Blood vessels were observed throughout the regenerated tissue. A layer of macrophages could be seen on the outer surface of the collagen guide channel.

At the $90^{\text {th }}$ day, the myelinated axon numbers were significantly greater for the collagen+CSF group $(5621 \pm 919)$ vs. the collagen+NS group $(3437 \pm 908)(p<0.05)$ (Figure 5). The average myelin sheath thickness for the autograft group $(0.41 \pm 0.18 \mu \mathrm{m})$ was greater compared to the collagen+CSF group $(0.49 \pm 0.21 \mu \mathrm{m})$, but the difference was not significant (p>0.05).

Immunoreactivity to S-100 was extensively observed in the cross section of the collagen+CSF group. Expression of the S-100 protein was located mainly in the myelin sheath in the collagen+CSF and autograft groups. There was distinctly more positive staining of the myelin sheath-associated protein S-100 in the collagen+CSF group. The Schwann cell existed around the myelinated axons (Figure 6A-D).

\section{DISCUSSION}

The findings of this study demonstrated that CSF in collagen guide channel significantly enhances peripheral nerve regeneration in vivo.

Table I: Comparison of NCV and AMP in Each Group at Days 30 and 90 Post Operation

\begin{tabular}{lcccc}
\hline \multirow{2}{*}{ Group } & \multicolumn{2}{c}{$\mathbf{N C V}(\mathbf{m} / \mathbf{S})$} & \multicolumn{1}{c}{ AMP (mV) } \\
\cline { 2 - 5 } & $\mathbf{3 0}^{\text {th }}$ & $\mathbf{9 0}^{\text {th }}$ & $\mathbf{3 0}^{\text {th }}$ & $\mathbf{9 0}^{\text {th }}$ \\
\hline Collagen + NS & $10.47 \pm 3.21$ & $21.23 \pm 4.51$ & $1.82 \pm 1.12$ & $3.63 \pm 1.42$ \\
\hline Collagen + CSF Autograft & $13.07 \pm 3.98$ & $39.7 \pm 5.92^{* *}$ & $2.71 \pm 1.68$ & $5.72 \pm 1.43$ \\
\hline Sham surgery & $12.6 \pm 3.45$ & $30.05 \pm 4.13$ & $2.57 \pm 0.68$ & $4.76 \pm 1.04$ \\
\hline
\end{tabular}

${ }^{*} P<0.05$, the difference between the sham surgery group and other experimental groups was significant. ${ }^{* *}$ The difference between the collagen+CSF group and the autograft and collagen + NS groups was significant $(P<0.05$, One-Way-ANOVA). Results are means $\pm S E M$.

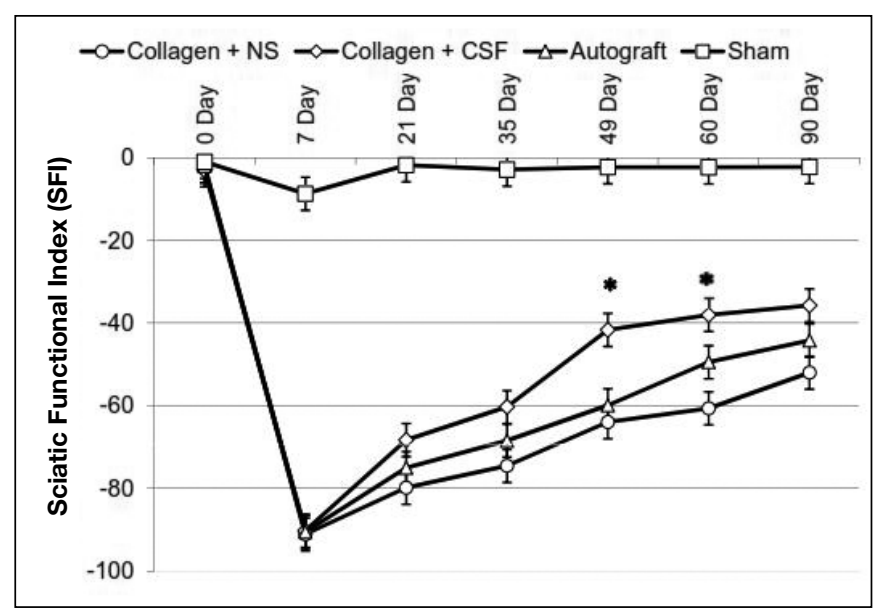

Figure 4: SFI before and after nerve injury in the collagen+CSF, collagen+NS, autograft, and sham surgery groups. "Difference between the experimental groups $(p<0.05$, $t$-test). Results presented as means \pm SEM.

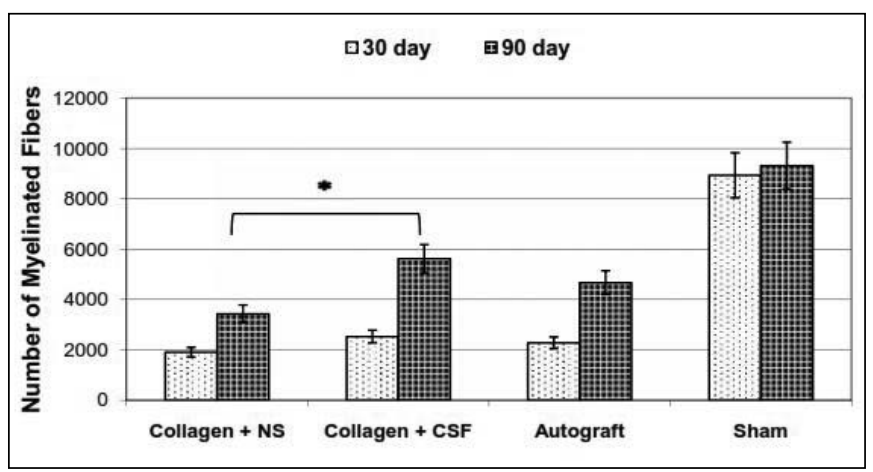

Figure 5: Total number of regenerated myelinated nerve fibers after sciatic injury ( $n=5$ on day 30 and $n=5$ on day 90 for each group). The difference between the collagen+CSF and collagen+NS groups at days 30 and 90 after operation, $(p<0.05$, One-Way-ANOVA). Results are presented as means \pm SEM. 
The collagen is a resorbable biomaterial for implant applications (1), and it is now widely used in both research environments and medical applications. The collagen nerve guide is flexible and strong enough to hold a suture and the dimensions of the guide are easily controlled (2), a secondary surgical site is not needed to obtain the collagen guide channel, and there is no donor site morbidity (1). In addition, the collagen can be obtained in large quantities, being inexpensive, sterilized and easily stored $(1,25,28)$. Collagen molecules can easily trap neurotrophic factors and collagen channels are able to adsorb cell adhesion molecules (17). Another advantage of using the collagen as a NGCs is that the degradation speed of this biological material can be controlled by manipulating the thickness of the guide channel (32).

Several studies have demonstrated that the collagen channel is as effective as a standard nerve autograft in terms of physiological recovery of target muscle activity $(2,33)$. Stang et al. reported that collagen does not seem to be a valuable material for an alternative nerve scaffold (26). The present study has shown, for the first time, that the rat CSF in collagen guide channel enhances peripheral nerve regeneration significantly more than the collagen guide channel without CSF in vivo. This was observed through a decrease of the SFI and increase of the myelin sheath thickness. It is well known that administration of exogenous neurotrophic factors after nerve injury has been shown to mimic the effect of target organ-derived trophic factors in neuronal cells (29).
The rat CSF contains numerous neurotrophic factors (nerve growth factor, fibroblast growth factor 2, ciliary neurotrophic factor) enhance peripheral nerve regeneration and neuroprotection (32). These factors may enhance the migration of Schwann cells into the guide channel, as well as proliferation, growth cone attachment on the guide channel wall and so regenerating axon elongation (17).

In our study, blood vessels were observed throughout the regeneration tissue in the collagen+CSF group. Chi et al. found that the adhesiveness of collagen for different cell type allows their long-term survival, proliferation, and angiogenesis (8). Yao et al. concluded that collagen may present a highly relevant biological microenvironment for axonal growth, and a range of collagen guide channels has been developed for nerve regeneration studies (33). The results from the above studies and our study suggest that rat CSF in collagen channel enhances peripheral nerve regeneration. Another possible explanation for these favorable effects could be the CSF ability to promote neurogenesis (32).

In our study, a layer of macrophages could be seen on the surface of the collagen guide channel. The macrophages were found on rough surfaces both in vitro (23) and in vivo (24) with evident signs of phagocytosis of the biomaterial. In addition, the macrophages also secrete a number of factors such as growth factors that can enhance healing, as well as promoters and regulators of inflammation and tissue degradation (7).

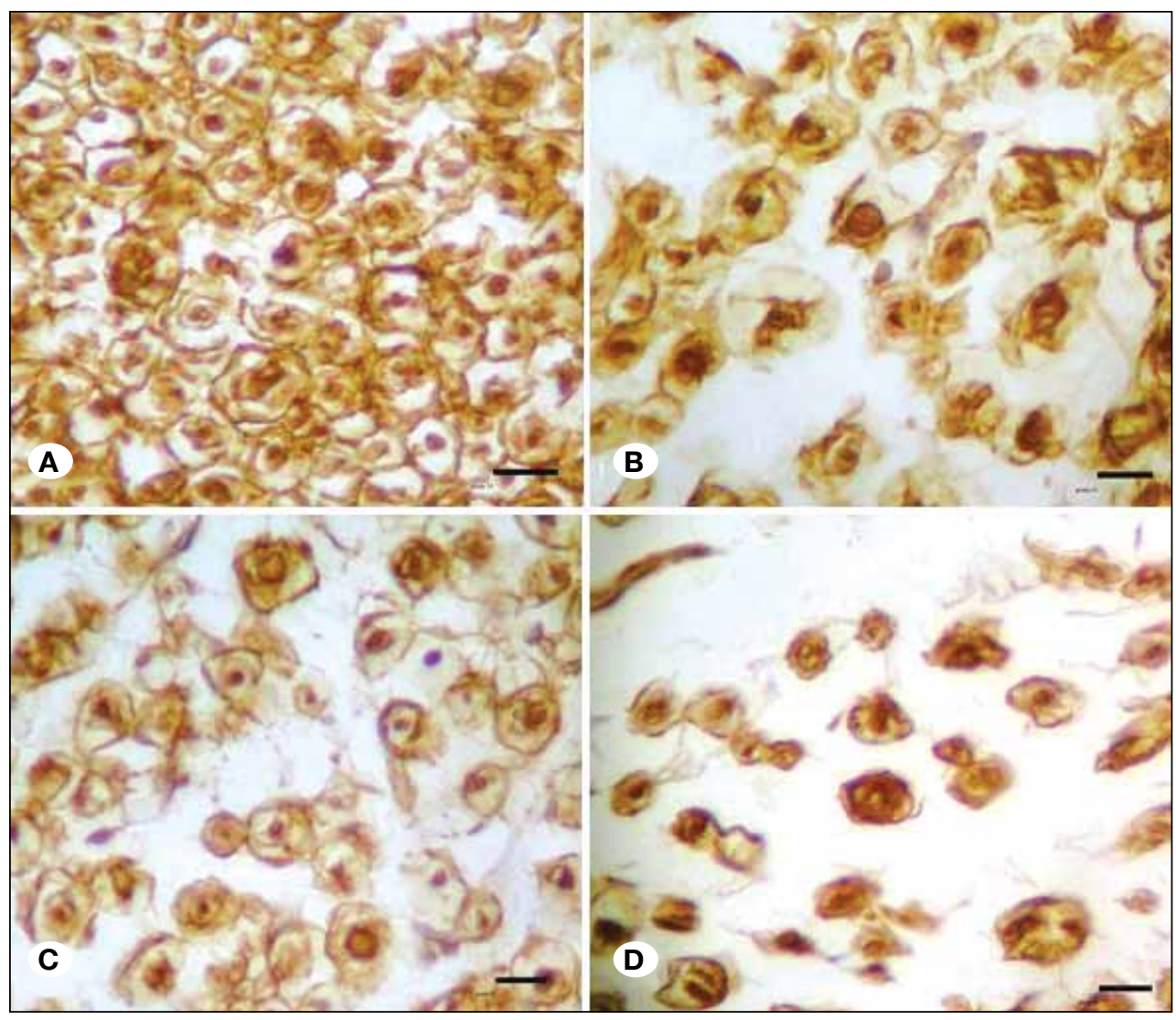

Figure 6: Immunohistochemical analysis of cross sections to the main axis of the regenerated nerve at day 90 after operation distal from the transected site of the sham surgery group (A), autograft group (B), collagen + CSF group (C), collagen + NS group (D). Positive staining of the myelin sheathassociated protein S-100 was observed. Regenerated nerve fibers containing Schwann cells, blood vessels, and myelinated axons were present throughout the tissue (scale bar $20 \mu \mathrm{m}$ ). 
In our study, there were no statistically significant differences between collagen+CSF and autograft SFI values at 90 days post-operation. The observation period was chosen to be 90 days, because the most of functional recovery occurred between 14 and 90 days post-operation (30), and muscle atrophy and joint stiffness were found after 10 weeks (8). It is to be noted that at 90 days post operation, animals maintained a $60 \%$ and a $80 \%$ deficit in NCV in autograft and collagen+CSF groups, respectively. The decreased NCV is one of the effect of the loss of larger diameter fibers, a phenomenon reported in other traumatic nerve lesions (10). There is an idea that the walking track analysis is more comprehensive and reliable than histomorphometric methods in peripheral nerve repair studies (14). This study supports the idea that the combination of SFI with electrophysiological assessment is more comprehensive than electrophysiological method alone (4). Nerve conduction velocity measures the fastest conducting nerve fibers, a measure that has been shown to be dependent on axon diameters, myelination, and internodal distance (23). A nerve may have a few fibers that conduct very well, even though a large number of remaining fibers are damaged (4).

\section{CONCLUSION}

CSF in collagen guide channels effectively enhances nerve regeneration, with a mechanism still to be explained. However, further studies are needed to evaluate the efficiency of autologus or artificial CSF in traumatic spinal cord injuries and other NGCs, such eggshell membrane, amnion layer, and chitosan.

\section{ACKNOWLEDGMENT}

This article was derived from the MSc thesis in the Urmia University of Medical Sciences. Funding for this research project was supported by grant no. 1103 from the Urmia University of Medical Sciences in Urmia, Iran.

\section{- REFERENCES}

1. Arakawa $\mathrm{Y}$, Sendtner $\mathrm{M}$, Thoenen $\mathrm{H}$ : Survival effect of ciliary neurotrophic factor (CNTF) on chick embryonic motoneurons in culture: Comparison with other neurotrophic factor and cytokines. J Neurosci 10(11): 3507-3515, 1990

2. Archibald SJ, Krarup C, Shefner J, Li ST, Madison RD: A collagen-based nerve guide conduit for peripheral nerve repair: An electrophysiological study of nerve regeneration in rodents and nonhuman primates. J Comp Neurol 306 (4): 685696, 1991

3. Bain JR, Mackinnon SE, Hunter DA: Functional evaluation of complete sciatic, peroneal and posterior tibial nerve lesions in the rat. Plast Reconstr Surg 83(1):129-138, 1989

4. Boccafoschi F, Habermehl J, Vesentini S, Mantovani D: Biological performances of collagen-based scaffolds for vascular tissue engineering. Biomaterials 26(35):7410-7417, 2005
5. Buddensiek J, Dressel A, Kowalski M, Storch A, Sabolek M: Adult cerebrospinal fluid inhibits neurogenesis, but facilitates gliogenesis from fetal rat neural stem cells. J Neurosci Res 87 (14):3054-3066, 2009

6. Cassar SC, Tovcimak AE, Rustay NR, Ellis TA, Hooker BA, Witte DG, Li J, Buck WR, Scharf D, Muller U, Jeromin A, Wang $\mathrm{KK}$, Waring JF: Comparing levels of biochemical markers in CSF from cannulated and non-cannulated rats. J Neurosci Methods 192(2):249-253, 2010

7. Chamberlain LJ, Yannas IV, Hsu HP, Strichartz G, Spector M: Collagen-GAG substrate enhances the quality of nerve regeneration through collagen tube up to level of autograft: Exp Neurol 154(2):315-329, 1998

8. Chi $\mathrm{H}$, Horie $\mathrm{H}$, Hikawa $\mathrm{N}$, Takenaka T: Isolation and agerelated characterization of mouse Schwann cells from dorsal root ganglion explants in type I collagen gels. J Neurosci Res 35(2):183-187, 1993

9. Chin CD, Khanna K, Sia SK: A microfabricated porous collagen-based scaffold as prototype for skin substitutes. Biomed Microdevices 10(3):459-467, 2008

10. Chvapil M, Kronenthal L, Van Winkle WJR: Medical and surgical applications of collagen. Int Rev Connect Tissue Res 6:1-61, 1973

11. Consiglio AR, Lucion AB: Technique for collecting cerebrospinal fluid in the cisterna magna of non-anesthetized rats. Brain Res Protoc 5(1):109-114, 2000

12. Farjah $G H$, Heshmatian $B$, Karimipour $M$, Saberi $A$ : Using eggshell membrane as nerve guide channels in peripheral nerve regeneration. Iran J Basic Med Sci 16(8):901-905, 2013

13. Gato A, Moro JA, Alonso MI, Bueno D, De La Mano A, Martin C: Embryonic cerebro-spinal fluid regulates neuroepithelial survival, proliferation, and neurogenesis in chick embryos. Anat Rec A Discov Mol Cell Evol Biol 284(1):475-484, 2005

14. Habermehl J, Skopinska J, Boccafoschi F, Sionkowska A, Kaczmarek $\mathrm{H}$, Laroche $\mathrm{G}$, Mantovani D: Preparation of a ready-to-use, stockable and reconstituted collagen. Macromol Biosci 5(9):821-828, 2005

15. Huang YC, Wang TW, Sun JS, Lin FH: Epidermal morphogenesis in an in-vitro model using a fibroblastembedded collagen scaffold. J Biomed Sci 12(6):855-867, 2005

16. Hudson TW, Evans GR, Schmidt CE: Engineering strategies for peripheral nerve repair. Clin Plast Surg 26(4):617-628, 1999

17. Itoh S, Takakuda K, Kawabata S, Aso Y, Kasai K, Itoh $\mathrm{H}$, Shinomiya K: Evaluation of cross-linking procedures of collagen tubes used in peripheral nerve repair. Biomaterials 23(23):4475-4481, 2002

18. Kim JK, Lee JS, Jung HJ, Cho JH, Heo JI, Chang YH: Preparation and properties of collagen/modified hyaluronic acid hydrogel for biomedical application. J Nanosci Nanotechnol 7(11):3852-3856, 2007

19. Kitahara A, Suzuki Y, Nishimura Y, Suzuki K, Kiyotani T, Takimoto Y, Nakamura T, Shimizu Y, Endo K: Evaluation of collagen nerve guide facial nerve regeneration. J Artif Organs 1:22-27, 1998 
20. Midha R, Shoichet MS, Dalton PD, Cao X, Munro CA, Noble J, Wong MK: Tissue engineered alternatives to nerve transplantation for repair of peripheral nervous system injuries. Transplant Proc 33(1-2):612-615, 2001

21. Miyan JA, Zendah M, Mashayekhi F, Owen-Lynch PJ: Cerebrospinal fluid supports viability and proliferation of cortical cells in vitro, mirroring in vivo development. Cerebrospinal Fluid Res 3(2):1-7, 2006

22. Mligiliche N, Endo K, Okamoto K, Fujimoto E, Ide C: Extracellular matrix of human amnion manufactured into tubes as conduits for peripheral nerve regeneration. J Biomed Mater Res 63(5):591-600, 2002

23. Rich A, Harris AK: Anomalous preferences of cultured macrophages for hydrophobic and roughened substrata. J Cell Sci 50:1-17, 1981

24. Salthouse TN: Some aspects of macrophage behavior at the implant interface. J Biomed Mater Res 18(4):395-401, 1984

25. Sionkowska A: The influence of UV light on collagen/poly (ethylene glycol) blends. Polymer Degradation and Stability 91:305-312, 2006

26. Stang F, Fansa H, Wolf G, Reppin M, Keilhoff G: Structural parameters of collagen nerve grafts influence peripheral nerve regeneration. Biomaterials 26(16):3083-3091, 2005

27. Stang F, Keilhoff G, Fansa H: Biocompatibility of different nerve tubes: Materials 2:1480-507, 2009

28. Szot CS, Buchanan CF, Freeman JW, Rylander MN: 3D in vitro bioengineered tumors based on collagen I hydrogels. Biomaterials 32(31):7905-7912, 2005
29. Terenghi G: Peripheral nerve regeneration and neurotrophic factors. J Anat 194(1):1-14, 1999

30. Tountas CP, Bergman RA, Lewis TW, Stone HE, Pyrek JD, Mendenhall HV: A comparison of peripheral nerve repair using an absorbable tubulization device and conventional suture in primates. J Appl Biomater 4(3):261-268, 1993

31. Verdu E, Labrador RO, Rodriguez FJ, Ceballos D, Fores J, Navarro $\mathrm{X}$ : Alignment of collagen and laminin-containing gels improve nerve regeneration within silicone tubes. Restor Neurol Neurosci 20(5):169-179, 2002

32. Wangensteen KJ, Kalliainen LK: Collagen tube conduits in peripheral nerve repair: A retrospective analysis. Hand (NY) 5(3):273-277, 2010

33. Yao L, de Ruiter GC, Wang H, Knight AM, Spinner RJ, Yaszemski MJ, Windebank AJ, Pandit A: Controlling dispersion of axonal regeneration using a multichannel collagen nerve conduit. Biomaterials 31(22):5789-5797, 2010

34. Yu W, Zhao W, Zhu C, Zhang X, Ye D, Zhang W, Zhou Y, Jiang $X$, Zhang Z: Sciatic nerve regeneration in rats by a promising electrospun collagen/poly ( $\varepsilon$-caprolactone) nerve conduit with tailored degradation rate. BMC Neurosci 12:68, 2011

35. Zappaterra MD, Lisgo SN, Lindsay S, Gygi SP, Walsh CA, Ballif BA: A comparative proteomic analysis of human and rat embryonic cerebro-spinal fluid. J Proteome Res 6(9):35373548, 2007

36. Zhao H, Ma L, Gao C, Shen J: Fabrication and properties of mineralized collagen chitosan/hydroxyapatite scaffolds. Polym Adv Technol 19:1590-1596, 2008 